\title{
Driving after total knee and hip arthroplasty - an observational study from the perspective of rehabilitative orthopaedic therapy
}

Tiberiu-Adrian Lazar ( $\square$ lazar.tiberiu@gmail.com )

Celenus Teufelsbad Fachklinik Blankenburg https://orcid.org/0000-0002-9094-5264

Martin Edelmann

Celenus Teufelsbad Fachklinik Blankenburg

Friedemann Awiszus

University Clinic Otto Von Guericke Magdeburg

Christoph Lohmann

Department of Orthopedic Surgery, Otto von Guericke University, Magdeburg

Research article

Keywords: Total knee arthroplasty, total hip arthroplasty, motor vehicle driving, rehabilitation

Posted Date: February 4th, 2021

DOl: https://doi.org/10.21203/rs.3.rs-168077/v1

License: (c) (i) This work is licensed under a Creative Commons Attribution 4.0 International License.

Read Full License 


\section{Abstract \\ Objective}

Considering the legal stipulations in Germany regarding fitness to drive a motor vehicle, the aim of this study is to find out when patients with a TKA or THA actually take up driving again after surgery; how the replaced joint, side, gender, place of residence and physician's recommendations, influences the patient in making the decision to start driving again.

\section{Background}

For a large proportion of the population, especially those residing in the countryside, the use of a car for daily activities is indispensable. Following a TKA or THA procedure, the overseeing physician will usually recommend refraining from driving, sometimes up to 12 weeks after surgery with a major social and economical impact on patient's life.

\section{Methods}

92 eligible participants, contained within the frame of a prospective experimental observational study, were contacted via telephone 12 weeks after surgery and interviewed using a structured questionnaire. The answers were statistically analysed using SPSS ${ }^{\circledR}$.

\section{Discussion}

There were statistically significant differences regarding operated side, gender and place of residence. Male patients drove in the 6th and 7th postoperative week, while female patients drove in the 8th and 9th postoperative week. For $58.6 \%$ of patients the reasoning for the first post-operative drive was medical: the journey to physiotherapy or to a doctor's appointment. TKA impaired patients the most. Patients recovering from a TKA drive considerably later. Patients recovering from a right sided TKA have an increased risk by a factor of nine, not to become an "early driver". Female patients who underwent TKA had an increased risk by a factor of 21 , of becoming a "late driver".

\section{Conclusions}

The rehabilitation following a right sided TKA proved a challenge with regard to the reuptake of driving. This should be taken into account when planning the course of therapy for patients who are regular drivers. Female patients could benefit from special training. 


\section{Trial registration:}

DRKS00018693. Registered 20 September 2019 https://www.drks.de/drks_web/navigate.do? navigationld=trial.HTML\&TRIAL_ID=DRKS00018693

\section{Background}

Arthrosis of the knee and hip joints is a common arthritic condition worldwide. In the GEDA 2014/2015EHIS study conducted by the Robert-Koch-Institute of Germany, $17.9 \%$ of adults above the age of 18 report having suffered from arthritis in the last 12 months.[1]

Replacement of the knee or hip joint (arthroplasty) following progressive joint destruction and function loss due to Osteoarthritis, has a major social and economical impact on patient's life. According to the Endoprosthesis Register in Germany (ERPD), approximately 450.000 endoprosthetic procedures (primary and revision arthroplasty) were carried out in 2018.[2] Also a search was run using the database of the German federal office of statistics (DESTATIS) on 24 January 2020 using the following string: "Koxarthrose/Gonarthrose" as a discharge diagnosis from hospital in 2018. It identified 178.329 patients with osteoarthrosis of the hip and 185.439 patients with osteoarthrosis of the knee.

Driving a car is important for people in general because it provides the opportunity for personal control and autonomy, especially for those residing in the countryside, due to a lack of public transport. For people having more difficulties walking or riding a bike due to health issues, driving a car is more than often the only option for a independent mobility, is often necessary to make doctor's and physiotherapy appointments and sometimes it is a requirement to resume working, not just in the remote countryside.

One of the most frequently asked questions, especially during the postoperative rehabilitation phase, by patients following a TKA or THA is "When can I start driving again?" In Germany, health care insurance providers are legally mandated (SGB IX, § 42) to finance a three-week in-patient postoperative rehabilitation (bedridden or ambulatory) following TKA or THA. Because most of the patients with a TKA or THA undergo a bedridden rehabilitation and they will be discharged between the 4th and 5th week after surgery, there is no reason to drive a motor vehicle before their discharge date. Also the legal stipulations in Germany require that every driver must evaluate whether they view themselves fit to drive prior to starting their engine. The driver must evaluate to what extent fulfils the requirement for roadworthiness. Doctor's recommendations should guide and support the patients in their evaluation. The decision to get behind the wheel of a motor vehicle, however, is made by the patient - that is what the legislation stipulates.

When driving, the ability to brake in time to avoid an obstacle is key. Both the cognitive and the motor skills are important. The interaction of these skills constitutes the brake reaction time, which represents a significant parameter of safe driving. That is why a large number of studies $[6,7,8,11,18]$ rightfully make use of this parameter. A brake reaction time within the normal range offers a decision-making tool 
for postoperatively reuptake of driving. The measured brake reaction time is a parameter of safe driving, however not the main criterion for postoperative roadworthiness.

Regarding recommendations when to reuptake driving after TKA or THA, there have been a lot of studies published. The authors of this paper have focused on the more recent published works and were able to find that there is a very large bandwidth of recommendations when to reuptake driving after TKA or THA, ranging from two days up to 8 weeks after surgery. $[4,5,11,12,13,14,15,16,17,18]$ In Germany, in some cases the recommendation to restrain from driving after TKA or THA procedures is 12 weeks after surgery. We've decided to take the 12 weeks timeframe as the maximum amount of recovery time after a

TKA or THA procedure, with no further complications, and set out to find to what extent patients take said recommendations into account when returning to motor vehicle driving after surgery.

Considering the legal stipulations in Germany regarding fitness to drive a motor vehicle, the aim of this study is to find out when patients with a TKA or THA actually take up driving again after surgery; how the replaced joint, side, gender, place of residence and physician's recommendations, influences the patient in making the decision when to start driving again.

\section{Methods}

The study design was that of a prospective experimental observational study, as a single center study in a orthopedic rehabilitation clinic in Germany.

For the statistical analysis the significance level was set for $5 \%$, while the confidence interval was set for $95 \%$. Pursuant to the concept of W. Viechtbauer et al., the ideal sample size was calculated to be of 90 participants.[3]

The study was approved by the ethics committee of Sachsen-Anhalt's medical board on the 11th of November 2019. Number 18/19.

Inclusion criteria: primary TKA or THA, age between 20 and 90 years, a valid driver's license, and regular driving practice. Exclusion criterion: total hip joint replacement surgery following trauma. Patient comorbidities were not taken into account.

In all, 100 participants consented, verbally and in written form, to the participation in the study, all of whom had been assigned to the Celenus Fachklinik "Teufelsbad" Blankenburg for in-patient rehabilitation from five surrounding hospitals. Recruitment occurred between the 17th of July 2019 and the 3rd of November 2019. In this time frame the authors oversaw the rehabilitation of 167 patients following TKA or THA. Of these, $59 \%$ were recruited into the study. This sample size fulfils criterion number 3 of the Quality Assessment Tool for Observational Cohort and Cross-Sectional Studies.[9]

The questionnaire used to collect the data was created specifically for this study and was based on the aim of this study. It included data about participants driving licence, age, sex, place of residence, operated 
joint and side, date of the operation, use of walking aids, driving practice, timeframe of postoperative driving resumption (weeks), pain level, car's transmission type and the reason for fist drive after surgery.

From the 13. post-operative week patients were contacted via telephone and the questionnaire was filled out by the caller, subject to the answers given by the participant. Hand written modifications were made in addition (placed of residence).

One participant revoked his consent, three other participants were no longer contactable via telephone. Four participants were wrongfully recruited (three participants with uni-compartmental knee replacement and one participant with total hip arthroplasties on both sides) and were excluded from the study. Finally, one participant had to be excluded as the data recording his place of residence was missing. Of the 100 original participants 92 remained who fulfilled the criteria and were included in the study. The drop-out rate was $4,1 \%$. (Flowchart patient enrolment)

The study sample (Table 1) consisted of 56 male and 36 female participants with an age range of between 34 and 81 years of age. The Mean age for male participants was 64.2 years of age, for female participants it was 60.6 years of age.

Table 1

Study sample

\begin{tabular}{|llll|}
\hline & Male & Female & Total \\
\hline Right TKA & 11 & 6 & $\mathbf{1 7}$ \\
\hline Left TKA & 14 & 11 & $\mathbf{2 5}$ \\
\hline Right THA & 16 & 10 & $\mathbf{2 6}$ \\
\hline Left THA & 15 & 9 & $\mathbf{2 4}$ \\
\hline Total & $\mathbf{5 6}$ & $\mathbf{3 6}$ & $\mathbf{9 2}$ \\
\hline
\end{tabular}

The statistical analyses of the collected data were conducted via the SPSS ${ }^{\circledR}$ Statistics software from IBM and Excel®. We assumed that the collected data about gender/operated joint/operated side/place of residence regarding the reuptake of driving had an equality of variances. Because of this assumption we had to use an ANOVA analysis, and furthermore several tests for two independent samples - Levene's Test for variance homogeneity, a t-Test, a Pearson Chi-Squared Test, Fischer's Exact Test and an Odds Ratio Analysis.

\section{Results}

92.4\% $(n=85)$ of the participants drove within the first 12 weeks of recovery following surgery. Seven participants refrained from driving - one participant suffered an apoplexy in the first 12 weeks post operation. Six participants answered the question "Did you resume driving after your operation?" with "I 
have not felt fit enough to drive (yet)". None of the participants who drove reported any accidents in the first 12 weeks of recovery.

$29.3 \%(n=27)$ of the participants resumed driving within the first 6 weeks after surgery. The earliest resumption of driving was within the 3rd postoperative week.

For $58.6 \%$ of the participants $(n=54)$ the first reason for driving was medical: driving to physiotherapy or a doctor's appointment.

The gender analysis of the postoperative resumption of driving showed that male participants resumed driving earlier than female participants $(p=0.0015)$. (Fig. 1)

The analysis of the operated side (left vs. right) showed a significant side preference $(p=0.019)$ in the postoperative resumption of driving. Participants who underwent a TKA or THA on the left side resumed driving earlier than participants who underwent a TKA or THA on the right side. (Fig. 2)

The Mode for the left side was 4th + 5th postoperative week, while the Mode for the right side was 8th + 9th postoperative week, with 17 and 14 participants, respectively.

The location of residence also had a statistically significant effect on the timeframe in which the participant resumed driving $(p=0.0299)$. Participants who lived in the countryside drove earlier than participants who lived in cities. (Fig. 3)

The Mode for participants who resided in the countryside is in the 4 th +5 th week post-surgery, while the Mode for participants who resided in cities is in the 6th +7 th week post-surgery, with 13 and 19 participants, respectively.

In our statistical analyses we were unable to find a significant difference $(p=0.053)$ between TKA and THA regarding the time frame in which the participants resumed driving.

The interaction between the operated side and operated joint was also analysed, the result of which showed a significance regarding TKA $(p=0.020)$ but no significance regarding THA $(p=0.304)$. Participants with left-sided TKA resumed driving earlier than participants with right-sided TKA. (Fig. 4) Additionally, we conducted two risk analyses. The risk analysis for side and joint presented statistical significance for TKA $(p=0.031)$. Regarding the Odds Ratio analysis for operated side, participants who were operated on the right side were 9.00 times less likely to be a "early driver" (resumption of driving before the 6 th +7 th week post-surgery; $95 \% \mathrm{Cl} 1.01-79.54)$. The risk analysis for gender and joint had also statistical significance $(p=0.00015)$; female participants who had undergone a TKA were 21.08 times more likely to be "late drivers" (resumption of driving 10th +11 th weeks post-surgery; $95 \% \mathrm{Cl} 3.64-$ 121.83). Therefore, female patients and patients who underwent a right-sided TKA can be considered high risk groups with regard to being a "late driver". 
Our assumption, that there would be a correlation between place of residence and reason for the first drive post-surgery could not be statistically supported $(p=0.927)$.

\section{Discussion}

This study showed that the recommendations for postoperative resumption of driving given by doctors and physical therapists were not heeded by patients. Rather, the participants resumed driving considerably earlier than recommended. On average male participants resumed driving between the 6th and 7th week post-surgery, female participants resumed driving between the 8th and 9th week postsurgery. This study aided in the verification of the fact that different study groups in part showcase drastically different results. For this reason, recommendations should be individualised as "one size fits all" recommendations are not helpful in this context.

A significant goal of regular driver who underwent a TKA or THA is the swift resumption of driving postsurgery. From a therapeutic perspective, this means that patients who wish to drive again shortly after surgery should receive individualised counsel and precise support so far as they are part of one of the risk groups mentioned above. By way of providing tailormade recommendations, the patient satisfaction can be positively influenced. This is important since the patient's subjective interpretation of success after knee or hip replacement surgery is heavily impacted by the fulfilment of the therapeutic expectations held by the patient.

A striking result of the gender analysis of postoperative driving was the binomial distribution of female participants. (Fig. 1) Male participants and a proportion of female participants behave similarly. A smaller proportion of female participants resumes driving later, in the 10th -12th week post-surgery.

Another result of the study was the discrepancy between participants who resided in the countryside and participants who resided in the city with regard to the timing of resumption following surgery. (Fig. 3) The authors defined "city" with a population of $\geq 30.000$ and "countryside" with a population of $<30.000$. This distinction is arbitrary, however, statistically, it showed significant differences with regard to when patients resumed driving post-surgery. The assumption is that "cities" boast a better transport infrastructure, which can be made use of and thus reduces the need for a car. Participants who reside in the "countryside" resumed driving considerably earlier (the Mode in the "countryside" was 13 participants in the 4th + 5th week post-surgery) than participants who reside in the "cities" (the Mode was 19 participants in the 6 th +7 th week post-surgery). $58.6 \%$ of the participants gave a medical reasoning for the first post-operative drive: a journey to a physical therapy appointment or a doctor's appointment. A hypothesised correlation between size of residential location and reason for the first post-operative drive could not be statistically confirmed $(p=0.927)$.

In a number of studies $[4,6,11,12,13,14]$ the operated side did not influence the postoperative resumption of driving. Our results showcase a different picture: there was no trend in patients who had undergone a THA $(p=0.304)$, in patients who had undergone a TKA, however, there was a trend of patients with a right-side arthroplasty driving significantly later $(p=0.020)$. A possible explanation for this 
is that driving requires little hip movement, whereas the right knee has the key function of pressing the gas and brake pedals. Thus, roadworthiness largely depends on the functionality and performance of the right knee. Clearly, this aspect is very central to the decision of roadworthiness the patient must make prior to driving.

In a study by Rondon et al. $98.2 \%$ of the study participants resumed driving within the first 12 weeks postsurgery.[5] We were able to deliver similar results, $92.4 \%$ of our study participants resumed driving in the first 12 weeks post-surgery.

In our study, the average time frame in which participants resumed driving following a TKA or THA was between the 6th and 7th week post-surgery. We were not able to find a significant difference between TKA and THA ( $p=0.053)$. By contrast, Rondon et al. discovered a statistically significant difference between knee and hip arthroplasties (TKA $=4.4$ weeks, $\mathrm{THA}=3.7$ weeks). [5]

This discrepancies could be explained by two possible reasons. In Germany, health insurance providers are legally mandated to finance a three-week in-patient postoperative rehabilitation following knee or hip arthroplasty. Our participants were recruited from the patient population in the rehabilitation facility and were therefore all undergoing a three-week in-patient rehabilitation, they were discharged between the 4th and 5th week post-surgery. For our study participants there was therefore no reason to drive before their discharge date. In addition to this, the sample size in this study was considerably smaller $(n=92)$ than the sample size in the study conducted by Rondon et al. $(n=1044)$.[5] This could explain why we were unable to find a statistically significant difference between total knee and hip arthroplasties with regard to the resumption of driving post-surgery. Rondon et al. identified the implementation of postoperative rehabilitation as a reason for later resumption of driving.[5]

We identified a higher risk of being a "late driver" if the participant underwent a right-side procedure or if the participant was female. Rondon et al. delivered similar results, there, too, patients whose right side was operated, and female patients drove later.[5] In addition, we conducted an Odds Ratio test with regard to the risk of resuming driving later post-surgery. An "early driver" was defined as a participant who resumed driving in the 4 th +5 th week post-surgery. A "late driver" was defined as a participant who resumed driving in the 10 th +11 th week post-surgery. There was a significantly $(p=0.031) 9.00$-fold $(95 \%$ $\mathrm{Cl} 1.01-79,54)$ increased risk for patients who had undergone a right-sided TKA not to resume driving in the 4 th +5 th week post-surgery. The risk analysis for gender and joint revealed a significant $(p=0.00015)$ 21.08-fold increased risk (95\% $\mathrm{Cl} 3.64-121.83)$ of female patients who had undergone TKA resuming driving in the 11 th +12 th week post-surgery. It therefore appears that female participants heed the recommendation given by doctors more than male participants. Consequently, this implies that female patients who have undergone a TKA should receive special guidance and a more intensive rehabilitation program if they intend to drive post-surgery. Of the female participants $13.9 \%(n=5)$ did not drive by the 13th week pot-surgery compared to $1.8 \%(n=1)$ of the male participants.

Davis et al. (2018) showed that both male and female participants $(n=32)$ achieve their preoperative brake reaction time after the 2 nd postoperative week.[6] Most of the participants drove with an automatic 
transmission. The operated side did not have an impact on how fast the preoperative brake reaction time was achieved. Gender, however, did: male participants reached their preoperative brake reaction earlier than female participants.[6]

In Germany, a driver must evaluate his own roadworthiness before he can start his engine.

Roadworthiness is defined as the ability to operate the vehicle safely, regardless of situation. Limitations of roadworthiness, for example drug or alcohol consumption are prosecuted by the legislator, however there are no legislative specifications regarding temporary physical limitations. The driver must evaluate himself in this regard and, provided he decides he is not roadworthy, refrain from driving. This selfevaluation is therefore crucial from a judicial standpoint. The present prospective study precisely examines this self-evaluation with regard to roadworthiness.

The Mean age of our male participants as 64.2 years, while the Mean age of our female participants was 60.6 years. The 65-74-year-old participants make up the group of drivers with the lowest accident rate at 21.5 accidents per 1000 drivers per year.[10] If extrapolated to the 3-month span, this would result in 5.4 accidents per 1000 drivers. Rondon et al. reported $0.9 \%$ accidents in the 3-month period for knee arthroplasties and $0.4 \%$ accidents in the 3-month period for hip arthroplasties. [5] A statistical comparison was not feasible since the accident rate was not elevated in drivers who had undergone TKA or THA when compared to the general age population. Our participants did not report any accidents. From this we can conclude that the self-evaluation of roadworthiness following TKA and THA was adequate and the individual time frame chosen by the participants was responsibly chosen.

After TKA or THA patients experience considerable limitations of the function of their affected leg for an uncertain period of time. This is why they turn to their overseeing doctor or physical therapist, for recommendations regarding post-op resumption of driving. A glance at the current research literature as an anchor or orientation regarding this recommendations, does not deliver consistent evidence.

The available studies can be categorised into two groups: studies which record when patients drive postsurgery and respect their participants' self-evaluations and studies which use measurable parameters, most often brake reaction time, to recommend when patients can resume driving post-surgery.

In the first group, roadworthiness is already reached two days following a minimally invasive THA (Quarashi et al.[11]), a week following a regular THA (Batra et al.[4]), two weeks following a regular THA (Van der Velden et al.[12]), 3.7 weeks following a regular THA (Rondon et al.[5]) and 6 th +7 th weeks postsurgery in the present study. The results for TKA are similarly inconsistent: 4 weeks post-surgery (Van der Velden et al.[12], 4.4 weeks post-surgery (Rondon et al.[5]), 6 weeks post-surgery [Ellanti et al.[13]), and in 6 th +7 th weeks post-surgery in the present study.

In a review study by Latz et al.[14] a recommendation of 2 to 4 weeks of rest is made before resumption of driving after a TKA or THA. Goodwin et al.[15], on the other hand, recommend 10 days to 8 weeks of rest following a right-sided TKA and 6 to 8 weeks of rest following a right-sided THA before resumption of driving. When it comes to the brake reaction time a similar picture is painted: Marques et al.[16] recorded 
$44 \pm 19$ days for the patients to reach their preoperative brake reaction time following a right-sided TKA and $20 \pm 15$ days following a left-sided TKA. Nizam et al.[17], however, recorded a Median of 3 weeks following a TKA, regardless of side. Van der Velden et al.[12] and Huang et al.[18] saw a return to the preoperative brake reaction time 4 weeks following a TKA and was able to show an inverse correlation between brake reaction time and the "step-test" by Marmon et al.[19].

To our knowledge, this study is one of the few that provides a unique point of view regarding the reuptake of driving after TKA or THA procedures, from the perspective of rehabilitative orthopaedic therapy. It showcases that the patient self-evaluation after a TKA or THA procedure is actually, at least in Germany, the main criterion for making the decision to reuptake driving again.

There were several limitations related to our study. Because most patients with a TKA or THA undergo a three week bedridden rehabilitation in Germany, there is until the end of the 5th postop week, no reason to reuptake driving again. It could be considered that the bedridden rehabilitation impairs the patient to reuptake driving earlier than they actually do. This is a particularity of the German health care system and could be considered a limitation of this study, when compared with other national health care systems, where a bedridden rehabilitation after a TKA or THA is not a common practice. Also the fact that this is a single center study and although the study sample size was calculated, with the help of a statistician, prior to study begin, it is too small to upscale this study results at the national level and only reflects the postoperative driving practices after TKA or THA of one region of Germany.

The large bandwidth of results across the board and the resulting variance of recommendations likely leave the patient at a loss. An operational approach which would examine the skills required for safe driving and base recommendations on these parameters would be more practical. A low pain level, sufficient range of motion (ROM) of the affected joint, unaided walking, safe entrance and exit from the vehicle, a preferably high score in the "step-test" and an acceptable brake reaction time seem to be the most crucial parameters to develop individual recommendations for patients. What role these parameters play in the decision-making process for the postoperative resumption of driving following a TKA or THA is unclear. Further research is required to examine the significance of these parameters in recommendations.

\section{Conclusions}

The key results of the present study are the significant differences of resumption of driving after surgery with regard to gender, place of residence, affected side and identification of high-risk groups.

When making the decision of when to resume driving following a TKA or THA patients should be advised individually. During the three-week in-patient rehabilitation program mandated in Germany patients should be supported and prepared, specifically female patients who have undergone TKA. The authors aim to develop a training program which can be implemented during the in-patient rehabilitation stay, with which the right knee can be trained to prepare for driving following a TKA. 


\section{Abbreviations}

TKA

total knee arthroplasty, THA-total hip arthroplasty.

\section{Declarations}

Ethics approval and consent to participate

The study was approved by the ethics committee "Ethikkommission der Ärztekammer Sachsen-Anhalt" of Sachsen-Anhalt's medical board on the 11th of November 2019. Number 18/19.

Written consent was obtained from the study participants.

Consent for publication

Not applicable

Availability of data and material

The datasets during and/or analysed during the current study are available from the corresponding author on reasonable request.

Competing interests

None declared.

Funding

No funding was obtained for this study.

Authors' contributions

All authors have read and approved the final manuscript.

TAL - study design and expose, literature research, study registration with the German registry for clinical studies, patient information and consent form design, ethics committee approval, patient recruitment, patient follow up, data management, writing and editing of the manuscript, graphics design.

ME - study design and expose, literature research, patient recruitment, writing and editing of the manuscript, publication journal research.

$\mathrm{CHL}$ - senior scientific advisor of the study, editing of the final manuscript.

FA - statistical analyses of raw data, graphics design. 
Acknowledgements

We would like to thank the chief physician Dr. med. Markus Appel, Celenus Fachklinik Teufelsbad Blankenburg for the support and guidance given for this study. We would also like to thank Miriam Edelmann for her assistance in translating the study into English.

\section{References}

1. Fuchs J, Kuhnert R, Scheidt-Nave C Journal of Health Monitoring · 2017 2(3) Robert Koch-Institut, Berlin, S. 55, doi 10.17886/RKI-GBE-2017-054.

2. Grimberg A, Jansson V, Melsheimer O, Steinbrück A. Endoprothesenregister Deutschland (ERPD) Jahresbericht 2019, S. -7. https://www.eprd.de/de/ueber-uns/aktuelles/artikel/eprd-jahresbericht2019/.

3. Viechtbauer W, Smits L, Kotz D, et al R. A simple formula for the calculation of sample size in pilot studies. J Clin Epidemiol. 2015;68(11):1375-9. doi.org/10.1016/j.jclinepi.2015.04.014.

4. Batra A, Gogos S, Nizam I. When do patients drive after minimally invasive anterior hip replacements? A single surgeon experience of 212 hip arthroplasties. SICOT-J 2018. 2018;4:51, 1-7.

5. Rondon AJ, Tan TL, Goswami K, et al. (2018) When Can I Drive? Predictors of Returning to Driving After Total Joint Arthroplasty, J Am Acad Orthop Surg 2020;28:427-433.

6. Davis JA, BohI DD, Gerlinger TL. Break response time after modern total knee arthroplasty: how soon can patients drive? Knee. 2018. doi.org/10.1016/j.knee.2018.07.002.

7. Pierson JL, Earles DR, Wood K. Brake response time after total knee arthroplasty: When is it safe to drive? J Arthroplasty Oct. 2003;18(7):840-3.

8. Ruel AV, Lee Y, Boles J, Boettner F, et al. A Novel Assessment of Braking Reaction time Following THA Using a New Fully Interactive Driving Simulator HSSJ (2015) 11:143-147.

9. Quality Assessment Tool for Observational Cohort and Cross-Sectional Studies. March 2014. Available online: https://www.nhlbi.nih.gov/health-topics/study-quality-assessment-tools (accessed on 01.06.2019).

10. Hamburger Abdenbaltt 15.7.2019 Anzahl den von PKW Fahrer verschuldeten Verkährsunfaälle pro 1000 Einwohner, Antwort des - Senats auf eine kleine Anfrage des CDU Verkherspolitikers Dennis Thering. https://www.abendblatt.de/hamburg/article226484339/Statistik-ergibt-Hamburgs-Seniorenfahren-am-sichersten.html (accessed on 30.06.2020).

11. Qurashi S, Chinnappa J, Lord SJ, et al. Driving after microinvasive total hip arthroplasty. J Arthroplasty. 2017;32:1525-9. https: \\doi.org/10.1016/j.arth.2016.11.052.

12. Van der Velden CA, Tolk JJ, Janssen RPA, et al. When is it safe to resume driving after total hip and knee arthroplasty? Bone Joint J. 2017;99-B:566-76. doi.org/10.1302/0301-620X.99B5.BJJ-20161064.R1. 
13. Ellanti P, Raval P, Harrington P. Return to driving after total knee arthroplasty. Acta Orthop Traumatol Turc. 2015;49:593-6.

14. Latz D, Schiffner E, Schneppendahl J, et al. Empfehlungen zur Fahrtauglichkeit bei Erkrankungen des Bewegungs- und Stütztaparates. Ein systematisches Review Unfallchirurg. 2019;122:697-705. doi.org/10.1007/s00113-018-0571-y.

15. Goodwin D, Baecher N, Pitta M, et al. Driving after orthopedic Surgery. Orthopedics. 2013 Jun;36(6):469-74. doi:10.3928/01477447-20130523-08.

16. Marques C, Barrerios J, Cabri J. What is the Evidence on Wich Physicians Can relay to advise patients when they may resume driving after TKA? A systemic literature review. J. Funct. Morphol. Kinesiol. 2018, 3,8.

17. Nizam I, Batra A, Gogs S. Early resumption of driving within 3 weeks following patient-specific instrumented total knee arthroplasty: an evaluation of 160 cases I. JISAKOS. 2019;4:88-92. doi:10.1136/jisakos-2018-000268.

18. Huang $\mathrm{H}$, Liang $\mathrm{J}$, Hung $\mathrm{W}$, et al. Timeframe for return to driving for patients with minimally invasive knee arthroplasty is associated with knee performance on functional tests. BMC Musculoskelet Disord. 2014;15:198. https://doi.org/10.1186/1471-2474-15-198.

19. Marmon A, McClelland J, Stevens-Lapsley J, et al. Single-step test for unilateral limb ability following total knee arthroplasty. J Orthop Sports Phys Ther. 2013;43(2):66-73.

\section{Figures}




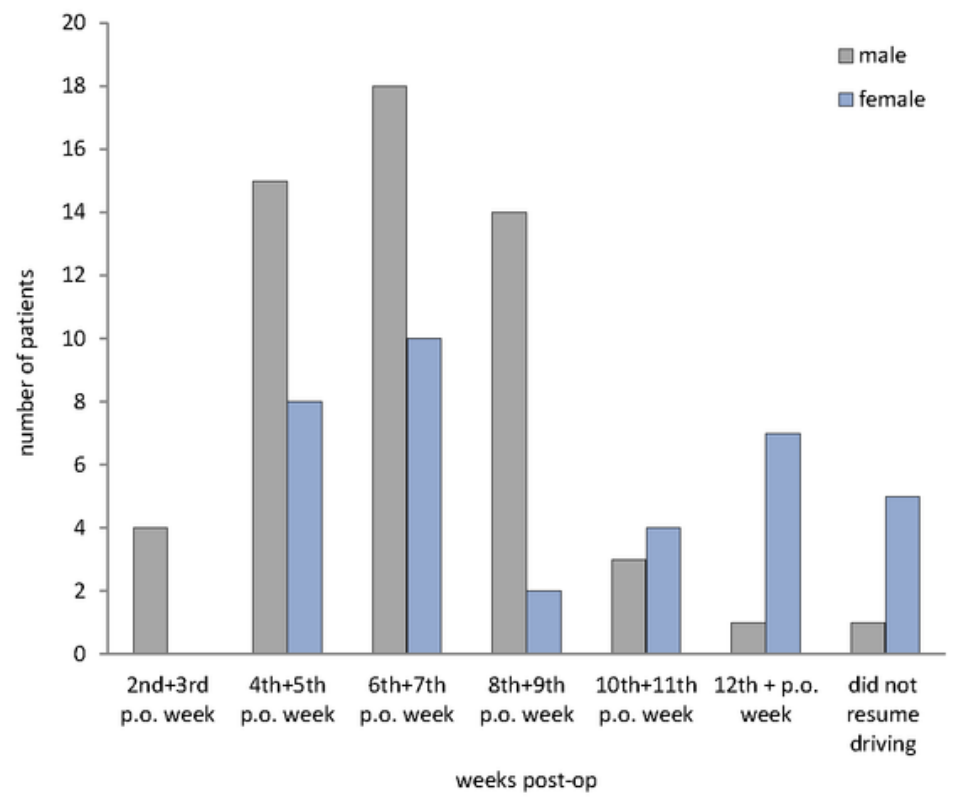

Figure 1

Resumption of driving after surgery depending on patient gender. $(n=92)$ 


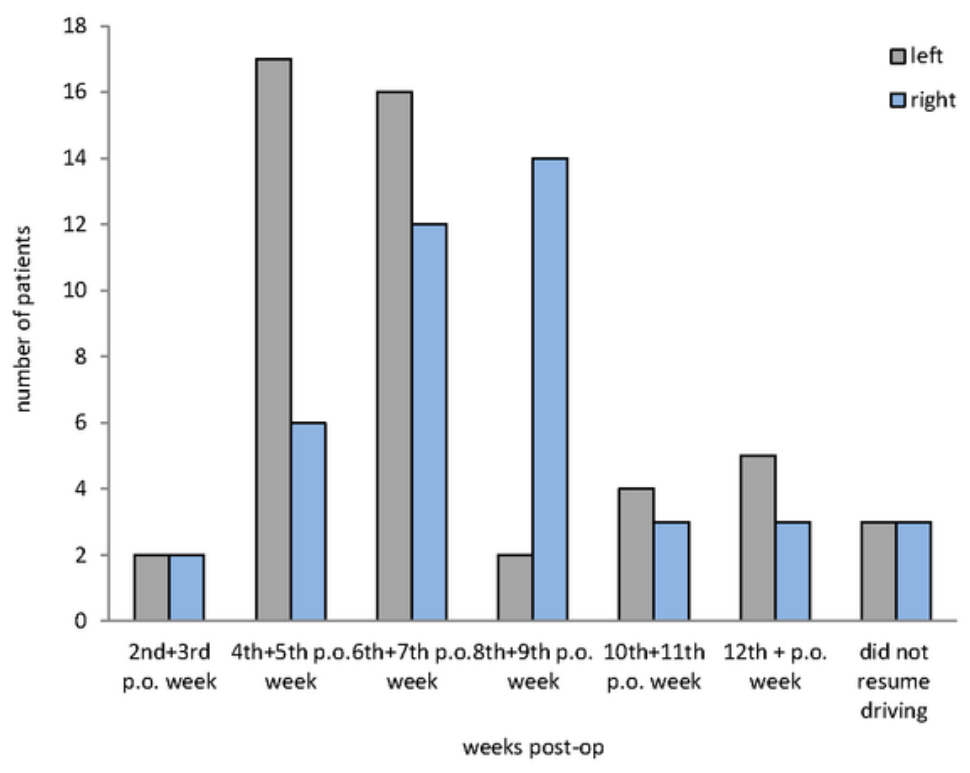

Figure 2

Resumption of driving after surgery depending on patient operated side. $(n=92)$ 


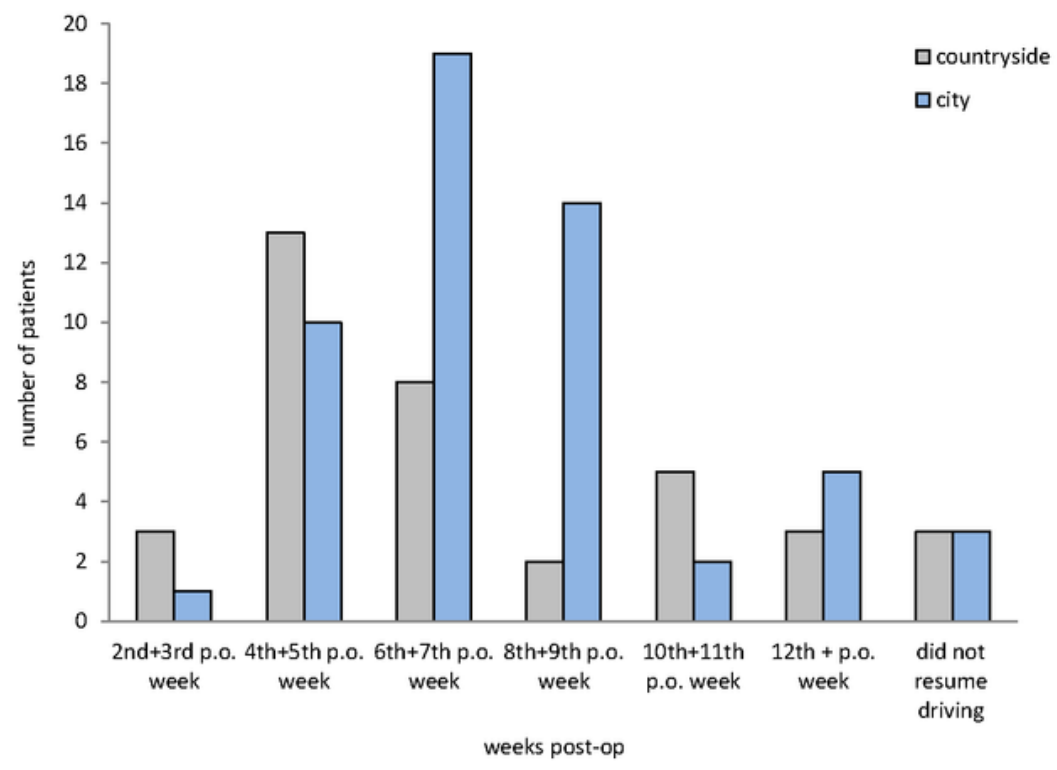

Figure 3

Resumption of driving after surgery depending on patient place of residence. ( $n=92$, countryside $<30.000$ residence; city $\geq 30.000$ residence) 
A total knee arthroplasty

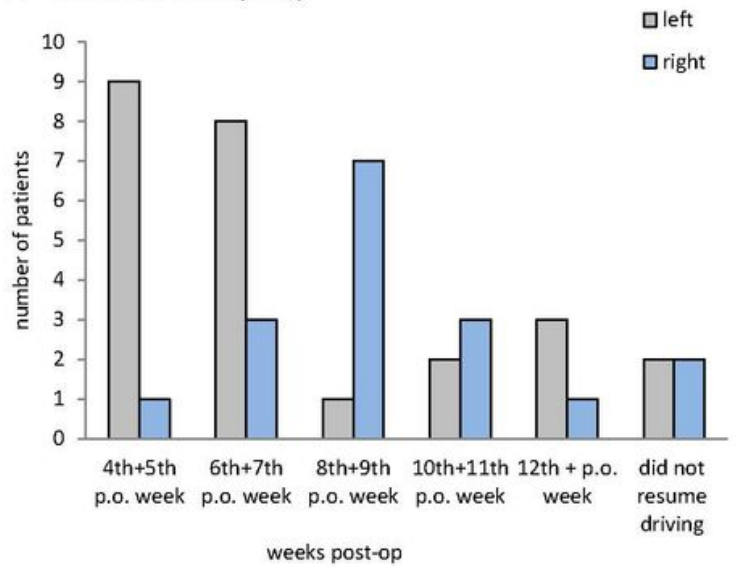

B total hip arthroplasty

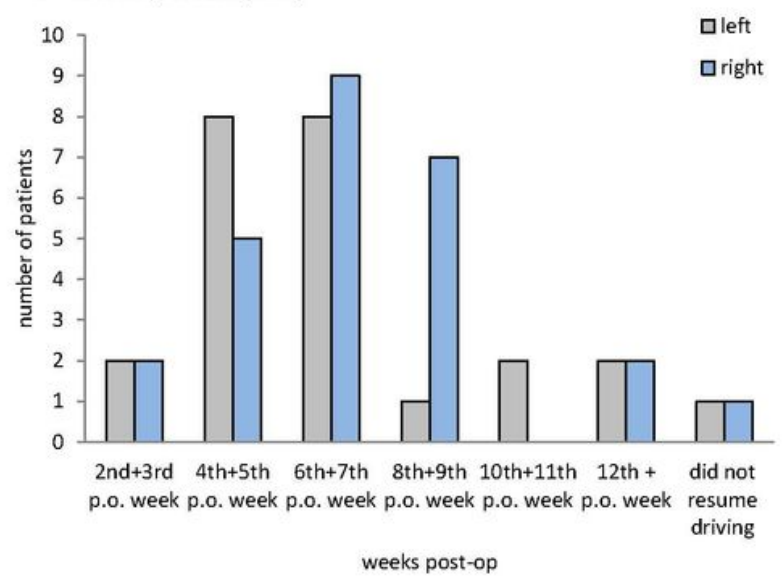

\section{Figure 4}

Interaction side/joint and resumption of driving after surgery. ( $n=92)$; A - total knee arthroplasty, B - total hip arthroplasty

\section{Supplementary Files}

This is a list of supplementary files associated with this preprint. Click to download.

- StudyQuestionnaire.pdf

- flowchartpatientrecruitement.docx 